\title{
Weakly Hyperbolic Actions of Kazhdan Groups on Tori
}

\author{
Benjamin Schmidt*
}

\begin{abstract}
We study the ergodic and rigidity properties of weakly hyperbolic actions. First, we establish ergodicity for $C^{2}$ volume preserving weakly hyperbolic group actions on closed manifolds. For the integral action generated by a single Anosov diffeomorphism this theorem is classical and originally due to Anosov.

Motivated by the Franks/Manning classification of Anosov diffeomorphisms on tori, we restrict our attention to weakly hyperbolic actions on the torus. When the acting group is a lattice subgroup of a semisimple Lie group with no compact factors and all (almost) simple factors of real rank at least two, we show that weak hyperbolicity in the original action implies weak hyperbolicity for the induced action on the fundamental group. As a corollary, we obtain that any such action on the torus is continuously semiconjugate to the affine action coming from the fundamental group via a map unique in the homotopy class of the identity. Under the additional assumption that some partially hyperbolic group element has quasi-isometrically embedded lifts of unstable leaves to the universal cover, we obtain a conjugacy, resulting in a continuous classification for these actions.
\end{abstract}

\section{Introduction}

In this article we investigate the notion of weak hyperbolicity for group actions first introduced in $\mathrm{MQ}$. For linear representations, weak hyperbolicity requires that there are no nontrivial subrepresentations for which all eigenvalues of all group elements have modulus one. Weak hyperbolicity for a group action on a closed manifold is a differential-geometric version of that for representations. The classical weakly hyperbolic dynamical systems, i.e. weakly hyperbolic actions of the integers, correspond to the well understood class of Anosov diffeomorphisms. With this in mind, we shall

*Partially funded by VIGRE grant DMS-9977371 
draw conclusions about weakly hyperbolic actions analogous to well known results describing Anosov diffeomorphisms. For example, Theorem 3.5 establishes the ergodicity of weakly hyperbolic actions, generalizing Anosov's work on ergodicity of Anosov diffeomorphisms [A, Theorem 4]. The proof of Theorem 3.5 uses ideas from the well known Hopf argument (see [BPSW, Section 2.1] for a description). Indeed, the proof is geometrically based on the presence (and accessibility) of stable foliations, and technically depends on the absolute continuity of these foliations. Perhaps new here is the fact that the core of this argument for ergodicity lies in the use of a regularity result from [RT] relating Sobolev classes of functions measured tangentially with respect to absolutely continuous foliations to global Sobolev classes of functions.

The remainder of this work is motivated by that of Franks and Manning $([\mathrm{F}]$, [Ma] ) on the classification of Anosov diffeomorphisms on tori. One may ask more generally whether weakly hyperbolic actions on tori are classifiable. When the acting group is a higher rank lattice, this question falls into Zimmer's program for classifying volume preserving ergodic actions of higher rank lattices on closed manifolds [Z]. Our Theorem 5.2 is an analogue of Manning's contribution to the classification of Anosov diffeomorphisms on tori. Roughly speaking, it asserts that weak hyperbolicity is inherited by the action on the fundamental group when the acting group is a higher rank lattice. The proof uses a rigidity property of the higher rank lattice, specifically that such groups have Kazhdan's property (T), to first draw conclusions about the action in the measurable category and then uses the dynamical assumption of weak hyperbolicity to bootstrap regularity from measurable to continuous. Theorem 5.2 confirms what is suggested to be true by Margulis and Qian in MQ. Therein, and more generally in [FW], the analogue of Frank's contribution is proven in the higher rank lattice setting. Their result coupled together with the complementary Theorem 5.2 establishes that all $C^{2}$ volume preserving weakly hyperbolic actions of higher rank lattices on tori, covered by an action on $\mathbb{R}^{n}$, are continuously semiconjugate to the affine action coming from the fundamental group (after possibly passing to a finite index subgroup of the lattice). If, in addition, some group element acts by a partially hyperbolic diffeomorphism with a quasi-isometric (in the universal cover) unstable foliation, then we show this semiconjugacy is injective, providing a continuous classification for this class of actions. We believe that the continuous semiconjugacy is injective without additional hypotheses and also believe a smooth classification should be possible.

We are glad to thank Jeffrey Rauch for providing Theorem 2.3 and outlining its Corollary 2.4, our advisor Ralf Spatzier, for his ongoing help and encouragement, and the referee for invaluable criticism on an earlier draft of this paper. 


\section{Background}

This section contains the definitions and results with relevance in subsequent sections.

\subsection{Partially Hyperbolic Dynamics}

Let $M$ be a closed Riemannian manifold and let $\mu$ denote the probability measure obtained by normalizing the Riemannian volume on $M$. An element $f \in \operatorname{Diff}^{k}(M)$ $(k \geq 1)$ is a partially hyperbolic diffeomorphism if there exist continuous $d f$-invariant subbundles $E_{f}^{s}, E_{f}^{c}, E_{f}^{u} \subset T M$, and real numbers $C \geq 1, a>b \geq 1$ such that:

- $T M=E_{f}^{s} \oplus E_{f}^{c} \oplus E_{f}^{u}$ and

- $\left\|d\left(f^{n}\right) v^{u}\right\| \geq C^{-1} a^{n}\left\|v^{u}\right\|,\left\|d\left(f^{n}\right) v^{s}\right\| \leq C a^{-n}\left\|v^{s}\right\|$, and $C^{-1} b^{-n}\left\|v^{c}\right\| \leq\left\|d\left(f^{n}\right) v^{c}\right\| \leq C b^{n}\left\|v^{c}\right\|$, for all $v^{u} \in E_{f}^{u}, v^{c} \in E_{f}^{c}, v^{s} \in E_{f}^{s}$ and all positive integers $n$.

We denote the set of $C^{k}$ volume preserving partially hyperbolic diffeomorphisms by $P H_{\mu}^{k}(M)$. The distributions $E_{f}^{s}$ and $E_{f}^{u}$ are called the strong stable and strong unstable distributions. It is well known (see [HPS, Theorem 5.5] or [P]) that these distributions integrate uniquely and that the resulting integral submanifolds are $C^{k}$ and form continuous foliations of $M$. These foliations will be denoted by $\mathcal{W}_{f}^{s}$ and $\mathcal{W}_{f}^{u}$ and are called the stable and unstable foliations. Recall that a d-dimensional continuous foliation $\mathcal{W}$ of $M^{n}$ by $C^{k}$ leaves is a partition of $M$ into locally immersed $C^{k} d$-dimensional submanifolds called leaves so that each point $x \in M$ has a foliated neighborhood, i.e a map $\Gamma: B^{d} \times B^{n-d} \rightarrow M$ where $B^{d}$ denotes the ball of dimension $d$ such that

- $\Gamma$ is a homeomorphism onto an open set in $M$ taking $(0,0)$ to $x$,

- for each $y \in B^{n-d}$, the map $\Gamma(\cdot, y): B^{d} \rightarrow M$ belongs to $C^{k}\left(B^{d}, M\right)$ and locally defines a leaf of the foliation, and

- the map $B^{n-d} \rightarrow C^{k}\left(B^{d}, M\right)$ given by $y \mapsto \Gamma(\cdot, y)$ is continuous in the $C^{k}$ topology.

The leaf through the point $x$ is denoted by $\mathcal{W}(\mathrm{x})$.

To mimic the classical Fubini theorem, one would like that the volume of a measurable set $E \subset M$ is obtained by integrating the volume of $E$ in leaves along a transversal to the foliation. A foliation $\mathcal{W}$ of $M$ is said to be absolutely continuous 
if for each open set $U$ of $M$ which is a union of local leaves and each local transversal $T$ to the foliation there is a measurable family of positive measurable functions $\delta_{x}: \mathcal{W}(x) \cap U \rightarrow \mathbb{R}$ so that for each measurable subset $E \subset U$,

$$
\mu(E)=\int_{T} \int_{W(x) \cap U} \chi_{E}(x, y) \delta_{x}(y) d \mu_{\mathcal{W}(x)}(y) d \mu_{T}(x),
$$

where $\mu_{\mathcal{W}(x)}$ and $\mu_{T}$ denote the Riemannian volumes in $\mathcal{W}(x)$ and $T$ respectively. Absolute continuity of a foliation as formulated above implies that zero volume subsets in the foliated manifold have zero volume in leaves through almost all points ([B1, Lemma 5.4]). Surprisingly, there are examples of foliations that are not absolutely continuous ([Mi]). A strictly stronger notion ([B1, Proposition 3.5]) is that of transversal absolute continuity of a foliation. To define this, first note that for any points $x_{1} \in M$ and $x_{2} \in \mathcal{W}\left(x_{1}\right)$ and choice of transversals $T_{i}$ to the foliation through the points $x_{i}(i=1,2)$, there is an associated Poincare map. This map is a homeomorphism $p: U_{1} \rightarrow U_{2}$ between neighborhoods $U_{i}$ of $x_{i}$ in $T_{i}$ satisfying $p\left(x_{1}\right)=x_{2}$ and $p(x) \in \mathcal{W}(x)$ for each $x \in U_{1}$. A foliation $\mathcal{W}$ is transversally absolutely continuous if all its Poincare maps are absolutely continuous maps with respect to the induced Riemannian measures on the transversals. In other words, for each choice of transversals $L_{1}$ and $L_{2}$ and associated Poincare map $p$, there is a positive measurable Jacobian $J: U_{1} \rightarrow \mathbb{R}$ such that for each measurable subset $A \subset U_{1}$,

$$
\mu_{T_{2}}(p(A))=\int_{U_{1}} \chi_{A}(x) J(x) d \mu_{T_{1}}(x) .
$$

If, in addition these Jacobians are continuous and positive, then the foliation is said to be measurewise $C^{1}$. Of technical importance is the following:

Theorem 2.1. [PS, Theorem 2.1] Let $f \in P H_{\mu}^{k}(M)$ and suppose $k \geq 2$. Then the stable and unstable foliations of $f$ are measurewise $C^{1}$.

In the course of the proof of Theorem 2.1, Pugh and Shub gave an asymptotic expression for the Jacobians of the Poincare maps. Starting from this expression, Nitica and Torok further strengthened the absolute continuity property for stable and unstable foliations. They studied the regularity properties of these foliations, first translating Pugh and Shub's work into a statement about the absolute continuity of the local coordinate charts for the foliation. Their formulation shows that Jacobians are differentiable along leaves and is useful for studying the regularity properties of functions on $M$ that restrict to regular functions on the leaves. The following follows from the proof of their regularity theorem: 
Theorem 2.2. [NT, Theorem 6.4] Let $f \in P H_{\mu}^{2}(M)$. Then there is a strong stable foliation chart $\Gamma: B^{d} \times B^{n-d} \rightarrow M$ around each $p \in M$ so that $\Gamma^{*}(\mu)=J(x, y) d x d y$ where the Jacobian $J$ is an everywhere positive and continuous function on $B^{d} \times B^{n-d}$ that has continuous (in $x$ and $y$ variables) partials of first order in the $x$ variables.

When a foliation $\mathcal{F}$ has local foliation charts as described in Theorem 2.2, we shall say the foliation $\mathcal{F}$ is strongly absolutely continuous. The next theorem is a local regularity result. It is used in subsequent sections to reduce proving continuity of a function on a manifold to proving that the function is Lipschitz when restricted to the stable leaves of a family of partially hyperbolic diffeomorphisms whose stable distributions jointly span the tangent bundle. Related results include $[\mathrm{HuK},[\mathrm{J}],[\mathrm{L}$, and [LMM]. First we establish some notation. For a function $u$ on $M$, a strongly absolutely continuous foliation $\mathcal{F}$, and $p \in(1, \infty)$, we say $u \in H_{\mathcal{F}}^{1, p}(M)$ provided that for each foliation chart as described by Theorem 2.2,

$$
\partial_{x_{i}}(u \circ \Gamma) \in L^{p}\left(B^{d} \times B^{n-d}\right), i=1, \ldots d,
$$

where differentiation is taken in the distributional sense. Recently, Rauch and Taylor have established that functions in $H_{\mathcal{F}}^{1, p}(M)$ are microlocally in the standard Sobolev space $H^{1, p}(M)$ away from the conormal bundle of $\mathcal{F}$ ([RT, Theorem 1.2]). This implies the following:

Theorem 2.3. [RT, Theorem 1.1] Let $\mathcal{F}_{1}, \ldots \mathcal{F}_{N}$ be strongly absolutely continuous foliations of $M$. Assume that for each $x \in M$,

$$
T_{x} M=\sum_{j=1}^{N} T_{x} \mathcal{F}_{j}
$$

Then, given $p \in(1, \infty)$, if $u \in H_{\mathcal{F}_{j}}^{1, p}(M)$ for each $j \in\{1, \ldots N\}$, then $u \in H^{1, p}(M)$, the standard Sobolev space.

Corollary 2.4. Let $\mathcal{F}_{1}, \ldots \mathcal{F}_{N}$ be strongly absolutely continuous foliations of $M$. Assume that for each $x \in M$,

$$
T_{x} M=\sum_{j=1}^{N} T_{x} \mathcal{F}_{j} .
$$

Suppose that $u \in L^{2}(M)$ and that there is a constant $K>0$ so that the restrictions of $u$ to almost all leaves of the foliations $\left\{\mathcal{F}_{j}\right\}$ are almost everywhere K-Lipshitz, then $u$ agrees almost everywhere with a continuous function. 
Proof. As a first step we argue that $u$ has bounded tangential first order derivatives parallel to the leaves of the foliations. These derivatives are only taken in the distributional sense. In a second step, we will use regularity bootstraping techniques to finish the proof. To this end, fix $j \in\{1, \ldots N\}, p \in M$, and let $d=\operatorname{dim} \mathcal{F}_{j}$. As the regularity properties of $u$ are a local matter, we may identify a local paramaterization of $\mathcal{F}_{j}$ in a neighborhood of $p \in M$ with a foliated open neighborhood $\Omega \subset \mathbb{R}^{n}$, described by a paramaterization

$$
\Gamma: U \times V \rightarrow \Omega
$$

with $U \subset \mathbb{R}^{d}, V \subset \mathbb{R}^{n-d}$ and Jacobian $J(x, y)$ as in Theorem 2.2. Let $X$ be a vector field in $\Omega$ such that $X \circ \Gamma=\sum_{j=1}^{d} a_{j}(x, y) \partial_{x_{j}}$, with $\partial_{x_{i}} a_{j}(x, y) \in C^{0}(U \times V)$ for $i=1, \ldots, d$ (such a field is well defined, independent of the choice of "flattening" paramaterization). Let $U^{\prime} \subset \subset U, V^{\prime} \subset \subset V$, denote by $\mathcal{D}^{\prime}\left(U^{\prime} \times V^{\prime}\right)$ the space of distributions on $U^{\prime} \times V^{\prime}$, and define

$$
X: L_{l o c}^{1}\left(U^{\prime} \times V^{\prime}\right) \rightarrow \mathcal{D}^{\prime}\left(U^{\prime} \times V^{\prime}\right),
$$

by $X(f)(\phi)=\int_{V^{\prime}} \int_{U^{\prime}} f\left(-\sum_{j=1}^{d} \partial_{x_{j}}\left(\phi a_{j}\right)\right) d x d y$ for $f \in L_{l o c}^{1}\left(U^{\prime} \times V^{\prime}\right)$ and $\phi \in C_{c}^{\infty}\left(U^{\prime} \times\right.$ $\left.V^{\prime}\right)$. Since $M$ has finite volume, $u \in L^{1}(M)$, and by continuity and positivity of the Jacobian $J$, it follows that $u \circ \Gamma \in L_{\text {loc }}^{1}\left(U^{\prime} \times V^{\prime}\right)$. To complete this step, we must show that $X(u \circ \Gamma) \in L^{\infty}\left(U^{\prime} \times V^{\prime}\right) \subset \mathcal{D}^{\prime}\left(U^{\prime} \times V^{\prime}\right)$. To this end we define (when $\mathrm{n}$ is sufficiently large) the difference quotients $u_{n, j}(x, y):=n\left(u \circ \Gamma\left(x+\frac{1}{n} e_{j}, y\right)-u \circ \Gamma(x, y)\right)$ and the functions $g_{n}:=\sum_{j=1}^{d} u_{n, j} a_{j}$. By the hypothesis on $u$ and the continuity of the $a_{j}$, each $g_{n} \in L^{\infty}\left(U^{\prime} \times V^{\prime}\right)$ and there is a uniform $C>0$ so that $\left\|g_{n}\right\|_{\infty}<C$. In particular there is a uniform bound $C^{\prime}$ on the $L^{2}$ norms of the $g_{n}$, so that a subsequence of the $g_{n}$ weakly converge to a function $g \in L^{2}\left(U^{\prime} \times V^{\prime}\right)$. This weak limit is also a distributional limit and furthermore it follows from the uniform bound $\left\|g_{n}\right\|_{\infty}<C$ that $\|g\|_{\infty}<C$. On the other hand, a routine change of variables argument shows that the $g_{n}$ converges to $X(u \circ \Gamma)$ in $\mathcal{D}^{\prime}\left(U^{\prime} \times V^{\prime}\right)$, completing this step.

By the first step and since $u \in L^{2}(M), u \in H_{\mathcal{F}_{j}}^{1,2}\left(M^{n}\right)$ for each $j \in\{1, \ldots, N\}$. Applying Theorem 2.3 establishes that $u \in H^{1,2}\left(M^{n}\right)$. By the Sobolev imbedding theorem (see [T, Proposition 2.2]), $H^{1, p}\left(M^{n}\right) \subset L^{\frac{n p}{n-p}}\left(M^{n}\right)$ for $p \in[1, n)$. From this, it follows that $u \in L^{\frac{2 n}{n-2}}\left(M^{n}\right)$. Again by the first step, we now obtain $u \in H_{\mathcal{F}_{j}}^{1, \frac{2 n}{n-2}}\left(M^{n}\right)$ for each $j$ and hence $u \in H^{1, \frac{2 n}{n-2}}\left(M^{n}\right)$ by Theorem 2.3. Note that for all $0<y<n$, $\frac{n y}{n-y}-y$ is positive and increasing as a function of $y$. We may therefore define a finite increasing sequence $\left\{x_{0}, \ldots x_{J}\right\}$ inductively by letting $x_{0}=2$ and $x_{i}=\frac{n x_{i-1}}{n-x_{i-1}}$ whenever $x_{i-1}<n$. Repeating the above argument shows that $u \in H^{1, x_{J}}\left(M^{n}\right)$. When 
$x_{J}=n$, note that $u \in H^{1, n-\epsilon}(M)$ for small $\epsilon>0$. Choose $\epsilon<\frac{n}{2}$ so that $\frac{n(n-\epsilon)}{n-(n-\epsilon)}>n$. Then $u \in H^{1, \frac{n(n-\epsilon)}{n-(n-\epsilon)}}(M)$. Therefore, $u \in H^{1, p}\left(M^{n}\right)$ for some $p>n$ whether $x_{J}>n$ or $x_{J}=n$. The conclusion of the corollary follows by the Sobolev imbedding (see [T, Proposition 2.4] $H^{1, p}\left(M^{n}\right) \subset C^{0}\left(M^{n}\right)$ for $p>n$.

\subsection{Kazhdan's property T}

Let $\Gamma$ be a discrete group and $\mathcal{H}$ a separable Hilbert space. A unitary representation $\pi: \Gamma \rightarrow U(\mathcal{H})$ is said to have almost invariant vectors if and only if for every $\epsilon>0$ and each compact subset $K \subset \Gamma$, there exists a unit vector $u(\epsilon, K) \in H$ so that $\|\pi(\gamma) u-u\|<\epsilon$ for each $\gamma \in K$. The group $\Gamma$ has Kazhdan's property (T) if every unitary representation of $\Gamma$ with almost invariant vectors has a nonzero invariant vector. It is well known that higher rank lattices have this fixed point property. There is a useful characterization of this property in terms of the vanishing of the first cohomology group of $\Gamma$ with coeficients in a unitary representation. Let $\phi$ be a representation of $\Gamma$ on a vector space $V$. A 1-cocycle is a map $c: \Gamma \rightarrow V$ such that $c(g h)=c(g)+\phi(g)(c(h))$ for each $g, h \in \Gamma$. A coboundary is a cocycle $c_{v}$ of the form $c_{v}(g)=v-\phi(g)(v)$ for some fixed $v \in V$. The first cohomology of $\Gamma$ with coeficients in the representation $\phi$ is the group of cocycles modulo the normal subgroup of coboundaries and is denoted by $H^{1}(\Gamma, \phi)$.

Theorem 2.5. $[D],[G]$ A group $\Gamma$ has Kazhdan's property if and only if $H^{1}(\Gamma, \phi)=0$ for every unitary representation $\phi$ of $\Gamma$.

\section{Weak Hyperbolicity, Accessibility, and Ergodic- ity}

The purpose of this section is to define the actions under consideration as well as the associated notion of accessibility. Recall that stable-unstable accessibility plays a central role in Hopf's argument for proving the ergodicity of Anosov systems. Here too, accessibility enters into a proof that $C^{2}$ weakly hyperbolic actions of discrete groups on closed manifolds are ergodic. In what follows, let $M$ denote a closed Riemannian manifold.

Definition. A weakly hyperbolic family on $M$ is a finite subset of $\mathrm{PH}_{\mu}^{1}(M)$ which is infinitesimally accessible in stable directions. More precisely, it is a finite family $\left\{\gamma_{1}, \ldots, \gamma_{k}\right\} \subset P H_{\mu}^{1}(M)$ with associated splittings $T M=E_{i}^{s} \oplus E_{i}^{c} \oplus E_{i}^{u}$ such that $T M=\sum_{i=1}^{k} E_{i}^{s}$. An action of a discrete group $\Gamma$ on $M$ is weakly hyperbolic if there is a finite family of group elements that act as a weakly hyperbolic family on $M$. 
The existence of the stable foliations suggests that infinitesimal accessibility along stable directions integrates to a notion of local accessibility along stable leaves.

Definition. Let $F=\left\{\gamma_{1}, \ldots, \gamma_{k}\right\}$ be a finite family of partially hyperbolic diffeomorphims on $M$ with associated stable foliations $\left\{\mathcal{W}_{1}^{s}, \ldots, \mathcal{W}_{k}^{s}\right\}$. An admissible path for $F$ is a path in $M$ that is piecewise sequentially contained in leaves of the stable foliations: i.e. a path $a:[0, T] \rightarrow M$ with a subdivision $t_{0}=0 \leq t_{1} \leq \cdots \leq t_{k}=T$ such that $a\left(\left[t_{j-1}, t_{j}\right]\right) \subset \mathcal{W}_{j}^{s}\left(a\left(t_{j-1}\right)\right)$ for each $j \in\{1, \cdots, k\}$. The family $F$ is locally accessible if for each $x \in M$ there is an open neighborhood $U_{x}$ of $x$ such that each $y \in U_{x}$ is the endpoint of an admissible path for $F$ beginning at $x$.

Lemma 3.1. A weakly hyperbolic family $F$ of partially hyperbolic diffeomorphisms on a closed manifold $M$ has the local accessibility property.

Proof. Let $F=\left\{\gamma_{1}, \ldots, \gamma_{k}\right\}$ be a weakly hyperbolic family on $M$ and fix $x \in M$. Note that any piecewise differentiable path in $M$ beginning at $x$ which is piecewise sequentially tangent to the stable distributions $\left\{E_{i}\right\}_{i=1}^{k}$ is an admissible path for $F$ since the stable distributions uniquely integrate. As this is a local question, we may view each stable distribution $E_{i}$ near $x$ as being given by the span of a finite ordered family of continuous vector fields $\left\{X_{i}^{1}, \ldots, X_{i}^{d(i)}\right\}$, where $d(i)=\operatorname{dim}\left(E_{i}\right)$. Any curve in $M$ which sequentially defines a solution to the fields $\left\{X_{i}^{j}\right\}_{j=1}^{d(i)}$ is tangent to $E_{i}$. Therefore, any curve that sequentially defines a solution to the combined ordered family of fields $X=\left\{X_{1}^{1}, \ldots, X_{1}^{d(1)}, \ldots, X_{k}^{1}, \ldots, X_{k}^{d(k)}\right\}$ is admissible for the family $F$. The hypothesis that the family $F$ is weakly hyperbolic implies that the family $X$ locally spans the tangent bundle near the point $x$. Establishing local accessibility for a weakly hyperbolic family therefore reduces to the next lemma.

Lemma 3.2. Let $\left\{X_{1}, \ldots, X_{N}\right\}$ be a family of nonvanishing continuous vector fields in $\mathbb{R}^{n}$ such that $\operatorname{span}\left(X_{1}, \ldots, X_{N}\right)=\mathbb{R}^{n}$. Then the set of endpoints of curves beginning at 0 which sequentially define solutions to these fields contains an open set around 0 .

In KP they study control systems with low regularity. Their Corollary 4.5 implies this last lemma. Note that if the vector fields had enough regularity to have $C^{1}$ exponential maps, then the spanning hypothesis implies that the composition of the exponential maps is a submersion at $0 \in \mathbb{R}^{n}$, from which the lemma follows. For continuous fields, solutions need not be unique and consequently, there are no exponential maps. The result in $[\mathrm{KP}]$ works in a significantly more general context and circumvents non-uniqueness by perturbing a system with low regularity to one with better regularity and applying a degree theory for set valued maps. An alternative approach is to approximate the family of continuous fields by a sequence of families 
of smooth fields and then argue that the sequence of open sets one obtains for the approximating families have a uniform lower bound on their size in order to take limits. For brevity, we omit the details of this direct approach.

In the remainder of this section we use local accessibility together with the regularity result, Corollary 2.4, in order to prove that $C^{2}$ weakly hyperbolic volume preserving actions of discrete groups are ergodic. Let $\Gamma$ be a discrete group. Recall that proving ergodicity for a measure preserving $\Gamma$ action on a Borel probability space $(X, \mu)$ is equivalent to showing that any square integrable function which is almost everywhere invariant under the action of $\Gamma$ is almost everywhere constant. To accomplish this, we first show that a square integrable function almost everywhere invariant under an element $\gamma \in P H_{\mu}^{2}(M)$ has the needed tangential regularity in order to apply Corollary 2.4. In fact, such a function will be essentially constant on almost all leaves of $\mathcal{W}_{\gamma}^{s}$. Weak hyperbolicity together with Corollary 2.4 then implies that each almost everywhere invariant representative of an $L^{2}$ element is necessarily equivalent to a continuous function $f$ which is almost everywhere invariant. As $f$ is continuous, it is everywhere invariant and hence constant on stable leaves. Local accessibility implies that $f$ is locally constant and therefore constant on $M$.

What follows makes this reasoning precise. Recall that for each volume preserving transformation $T$ of $M, \mu$ has an ergodic decomposition ([FMW, Theorem 2.19]); more precisely, there is a full volume $T$-invariant Borel set $M^{\prime} \subset M$, a standard Borel probability space $(\Omega, \nu)$, a Borel map $\xi: \Omega \rightarrow \operatorname{Prob}\left(M^{\prime}\right)$, and a $T$-invariant Borel map $\psi: M^{\prime} \rightarrow \Omega$ such that $\xi(\omega)\left(\psi^{-1}(\omega)\right)=1$ for each $\omega \in \Omega, \mu=\int_{\Omega} \xi(\omega) d \nu(\omega)$, and $\xi(\omega)$ is quasi-invariant and ergodic for each $\omega \in \Omega$, where $\operatorname{Prob}\left(M^{\prime}\right)$ inherits its Borel structure from the weak* topology.

Lemma 3.3. Suppose that $T \in P H_{\mu}^{1}(M)$ has an absolutely continuous stable foliation and that a square integrable function $f$ is almost everywhere $T$-invariant. Then for almost all leaves, $f$ restricted to the leaf is almost everywhere constant.

Proof. For $g \in L^{1}(M, \mu)$ we denote the set of Birkhoff regular points for $g$ by $B_{g}:=$ $\left\{x \in M^{\prime} \mid g^{+}(x)=\int_{M^{\prime}} g d \xi(\psi(x))\right\}$, where $g^{+}(x)$ is given by $\lim _{n \rightarrow \infty} \frac{1}{n} \sum_{i=0}^{n-1} g\left(T^{i}(x)\right)$ at points where this limit converges. It follows easily from the description of the ergodic decomposition that these sets are Borel sets and the Birkhoff ergodic theorem implies these sets have full volume in $M$. From the separability of $C^{0}(M)$, it follows that the set $B_{0}:=\cap_{g \in C^{0}(M)} B_{g}$ has full volume in $M$. By hypothesis there is a full volume $T$-invariant set $I \subset M$ so that $f$ is $T$-invariant in $I$. By absolute continuity of the stable foliation, there is a full volume subset of good points $G \subset\left(I \cap B_{f} \cap B_{0}\right)$ such that $x \in G$ implies $\left(I \cap B_{f} \cap B_{0}\right)$ is conull in $\mathcal{W}_{T}^{s}(x)$. Fix $x \in G$ and let $y_{1}, y_{2} \in \mathcal{W}_{T}^{s}(x) \cap\left(I \cap B_{f} \cap B_{0}\right)$. We first argue that $y_{1}$ and $y_{2}$ lie in the same ergodic component, or more precisely, that $\xi\left(\psi\left(y_{1}\right)\right)=\xi\left(\psi\left(y_{2}\right)\right)$. Indeed the Hopf 
argument (see e.g. BPSW, Section 2.1]) shows that for any continuous function $g$, whenever $g^{+}(x):=\lim _{n \rightarrow \infty} \frac{1}{n} \sum_{i=0}^{n-1} g\left(T^{i}(x)\right)$ converges, $g^{+}(y)$ converges to $g^{+}(x)$ for all $y \in \mathcal{W}_{T}^{s}(x)$. Since $y_{1}, y_{2} \in B_{0}$, it follows that $\int_{M} g d \xi\left(\psi\left(y_{1}\right)\right)=\int_{M} g d \xi\left(\psi\left(y_{2}\right)\right)$ for all continuous functions $g$, whence $\xi\left(\psi\left(y_{1}\right)\right)=\xi\left(\psi\left(y_{2}\right)\right)$. Since $y_{1}, y_{2} \in\left(I \cap B_{f}\right)$,

$$
f\left(y_{1}\right)=f^{+}\left(y_{1}\right)=\int_{M^{\prime}} f d \xi\left(\psi\left(y_{1}\right)\right)=\int_{M^{\prime}} f d \xi\left(\psi\left(y_{2}\right)\right)=f^{+}\left(y_{2}\right)=f\left(y_{2}\right) .
$$

From Corollary 2.4 and Lemma 3.3 we deduce the following:

Corollary 3.4. Suppose that $f$ is a square integrable function on $M$ that is almost everywhere invariant under a weakly hyperbolic $C^{2}$ family on $M$. Then $f$ is almost everywhere equal to a continuous function $g$.

Theorem 3.5. Let $\rho$ be a $C^{2}$ volume preserving weakly hyperbolic action of a discrete group $\Gamma$ on $M$. Then the $\Gamma$ action is ergodic.

Proof. Let $f$ be any square integrable almost everywhere $\Gamma$-invariant function. By the last corollary, $f$ is almost everywhere equal to a continuous function $g$ that is almost everywhere $\Gamma$-invariant. By continuity of $g$ and since full volume sets are dense, $g$ is everywhere invariant. Hence $g$ is constant on all stable leaves of all the elements in the weakly hyperbolic family. By local accessibility, $g$ is locally constant and hence constant. Therefore $f$ is essentially constant.

\section{Weak Hyperbolicity is Inherited}

In this section we consider volume preserving weakly hyperbolic actions of discrete property $(\mathrm{T})$ groups $\Gamma$ on tori. We argue that whenever such an action is covered by a $\Gamma$ action on $\mathbb{R}^{n}$, the representation coming from the homomorphism $\Gamma \rightarrow \operatorname{Out}\left(\pi_{1}\left(\mathbb{T}^{n}\right)\right)$ cannot split as a nontrivial direct sum of subrepresentations, one of which is isometric. To this end, let $\Gamma$ denote a discrete Kazhdan group and $\rho: \Gamma \rightarrow \operatorname{Diff}^{2}\left(\mathbb{T}^{n}\right)$ be a volume preserving weakly hyperbolic action covered by an action, $\bar{\rho}: \Gamma \rightarrow \operatorname{Diff}^{2}\left(\mathbb{R}^{n}\right)$. The action $\rho$ induces a homomorphism $\Gamma \rightarrow \operatorname{Out}\left(\pi_{1}\left(\mathbb{T}^{n}\right)\right)$ that lifts to a homomorphism $\pi: \Gamma \rightarrow \operatorname{Aut}\left(\mathbb{Z}^{n}\right)$. Note that $\pi$ satisfies

$$
\bar{\rho}(\gamma)(x+z)=\bar{\rho}(\gamma)(x)+\pi(\gamma)(z),
$$

for each $\gamma \in \Gamma, x \in \mathbb{R}^{n}$, and $z \in \mathbb{Z}^{n}$. For simplicity, we also let $\pi$ denote the representation $\Gamma \rightarrow \operatorname{GL}\left(n, \mathbb{R}^{n}\right)$ induced by the homomorphism $\pi$. Finally, let $\rho_{0}$ denote the linear action on the torus induced by $\pi$. We assume that there is a direct 
sum decomposition of $\mathbb{R}^{n}$ into $\Gamma$ invariant subspaces $C$ and $H$ so that the restriction of $\pi$ to $C, \pi^{C}$, acts isometrically in $C$ and will argue that $C=\{0\}$. The main idea is that the representation $\pi$ coarsely approximates the lifted action $\bar{\rho}$. Since all tangent directions are spanned by directions that are contracted uniformly by some element of the group under $\bar{\rho}$, the approximating action $\pi$ cannot have a nontrivial isometric invariant subspace $C$. Making this line of reasoning precise involves analyzing a cocycle (first introduced in $\mathrm{MQ}$ ) which measures the difference between the lifted action and the induced action on the fundamental group. The heart of the argument lies in the following:

Proposition 4.1. Assume the hypotheses above. Then there is a continuous map $\phi: \mathbb{R}^{n} \rightarrow C$ of the form $\phi(x)=\operatorname{proj}_{C}(x)+\sigma([x])$ where $\sigma \in C^{0}\left(\mathbb{T}^{n}, C\right)$ such that $\pi^{C}(\gamma)(\phi(x))=\phi(\bar{\rho}(\gamma) x)$.

Proof. Define the map $A: \Gamma \times \mathbb{R}^{n} \rightarrow \mathbb{R}^{n}$ by the equation

$$
\bar{\rho}(\gamma) x=\pi(\gamma)(x+A(\gamma, x)) .
$$

In view of how $\pi$ is defined, for a fixed $\gamma \in \Gamma$, the function $A(\gamma, \cdot)$ descends to a function on the torus. Following $A$ by the projection to $C$ parallel to the complementary subspace $H$, we obtain a map $A^{C}: \Gamma \times \mathbb{T}^{n} \rightarrow C$. Since $H$ is an invariant complement to $C, A^{C}$ solves the equation

$$
\operatorname{proj}_{C}(\bar{\rho}(\gamma) x)=\pi^{C}(\gamma)\left(\operatorname{proj}_{C}(x)+A^{C}(\gamma,[x])\right) .
$$

Define the unitary representation $\lambda: \Gamma \rightarrow U\left(L^{2}\left(\mathbb{T}^{n}, C\right)\right)$ by

$$
(\lambda(\gamma) f)([x]):=\pi^{C}(\gamma) f\left(\rho\left(\gamma^{-1}\right)[x]\right) .
$$

Next, we check that the map $c: \Gamma \rightarrow L^{2}\left(\mathbb{T}^{n}, C\right)$ given by $\gamma \mapsto A^{C}\left(\gamma^{-1}\right)$ is a 1-cocycle in $Z^{1}(\Gamma, \lambda)$. As a preliminary step, we establish

$$
(*) A^{C}\left(\gamma_{1} \gamma_{2},[x]\right)=A^{C}\left(\gamma_{2},[x]\right)+\pi^{C}\left(\gamma_{2}^{-1}\right) A^{C}\left(\gamma_{1}, \rho\left(\gamma_{2}\right)[x]\right) .
$$

Indeed,

$$
\begin{gathered}
\pi\left(\gamma_{1} \gamma_{2}\right)\left(x+A\left(\gamma_{1} \gamma_{2},[x]\right)\right)=\bar{\rho}\left(\gamma_{1}\right)\left(\bar{\rho}\left(\gamma_{2}\right) x\right)=\pi\left(\gamma_{1}\right)\left(\bar{\rho}\left(\gamma_{2}\right) x+A\left(\gamma_{1}, \rho\left(\gamma_{2}\right)[x]\right)\right)= \\
\pi\left(\gamma_{1}\right)\left[\pi\left(\gamma_{2}\right)\left(x+A\left(\gamma_{2},[x]\right)\right)+A\left(\gamma_{1}, \rho\left(\gamma_{2}\right)[x]\right)\right]= \\
\pi\left(\gamma_{1} \gamma_{2}\right)\left(x+A\left(\gamma_{2},[x]\right)+\pi\left(\gamma_{2}^{-1}\right) A\left(\gamma_{1}, \rho\left(\gamma_{2}\right)[x]\right)\right) .
\end{gathered}
$$

Applying $\pi\left(\gamma_{2}^{-1} \gamma_{1}^{-1}\right)$ to the first and last term and projecting to the subspace $C$ gives $(*)$. Therefore,

$A^{C}\left(\gamma_{2}^{-1} \gamma_{1}^{-1}, \cdot\right)=A^{C}\left(\gamma_{1}^{-1}, \cdot\right)+\pi^{C}\left(\gamma_{1}\right) A^{C}\left(\gamma_{2}^{-1}, \rho\left(\gamma_{1}^{-1}\right) \cdot\right)=A^{C}\left(\gamma_{1}^{-1}, \cdot\right)+\lambda\left(\gamma_{1}\right) A^{C}\left(\gamma_{2}^{-1}, \cdot\right)$, 
showing $c \in Z^{1}(\Gamma, \lambda)$.

By Theorem 2.5 there is some $\sigma \in L^{2}\left(\mathbb{T}^{n}, C\right)$ such that

$$
(* *) A^{C}\left(\gamma^{-1}\right)=\sigma-\lambda(\gamma) \sigma,
$$

holds as an equation in $L^{2}$ for every $\gamma \in \Gamma$. Next, we argue that $\sigma$ agrees almost everywhere with a continuous function, and consequently, that $(* *)$ holds as an equation in $C^{0}\left(\mathbb{T}^{n}, C\right)$. In view of Corollary 2.4 , it suffices to show that for a partially hyperbolic diffeomorphism $\rho(\gamma) \in P H_{\mu}^{2}\left(\mathbb{T}^{n}\right)$, there is a uniform constant $C>0$ such that the restriction of $\sigma$ to almost all leaves of the stable foliation $\mathcal{W}_{\rho(\gamma)}^{s}$ is almost everywhere $C$-Lipschitz. To show this, we first simplify notation writing $\gamma$ instead of $\rho(\gamma), \hat{\gamma}$ instead of $\pi^{C}\left(\gamma^{-1}\right)$, and will let $f$ denote the $C^{2}$ function $A^{C}(\gamma)$. In this simplified notation, $(* *)$ implies that there is a full volume $\gamma$-invariant set $I \subset \mathbb{T}^{n}$ so that

$$
(* * *) \sigma(x)=f(x)+{ }^{\hat{\gamma}} \sigma\left({ }^{\gamma} x\right)
$$

for $x \in I$. By the ergodic decomposition ([FMW, Theorem 2.19]), there is a full volume $\gamma$-invariant set $M^{\prime} \subset \mathbb{T}^{n}$, a standard Borel probability space $(\Omega, \nu)$, a $\gamma$ invariant Borel map $\psi: M^{\prime} \rightarrow \Omega$, and a Borel map $\xi: \Omega \rightarrow \operatorname{Prob}\left(M^{\prime}\right)$ such that $\mu=\int_{\Omega} \xi(\omega) d \nu$, where each $\xi(\omega)$ is a quasi-invariant ergodic probability measure. As in Lemma 3.3, for each $g \in L^{1}$ define the full volume set $B_{g} \subset M^{\prime}$ by $B_{g}:=$ $\left\{x \in M^{\prime} \mid g^{+}(x)=\int_{M^{\prime}} g d \xi(\psi(x))\right\}$ and the full volume set $B_{0}=\cap_{g \in C^{0}} B_{g}$. By Lusin's theorem there is a sequence of compact sets $K_{j} \subset K_{j+1}$ such that the restriction of $\sigma$ to $K_{j}$ is uniformly continuous and $\mu\left(K_{j}\right)>1-\frac{1}{2^{j}}$. Let $K=\bigcup_{j} K_{j}$ and $B_{K}:=\left\{x \in M^{\prime} \mid \xi(\psi(x))(K)=1\right\}$. It is straightforward to argue that $B_{K}$ is a full volume Borel set. Finally, let $G:=I \cap B_{K} \cap_{j} B_{\chi\left(K_{j}\right)} \cap B_{0}$. Then $G$ is a full volume Borel set and by absolute continuity, there is a full volume subset of points $E \subset G$ so that $G$ is conull in $\mathcal{W}_{\gamma}^{s}(x)$ whenever $x \in E$. Fix $x \in E$ and $y_{1}, y_{2}$ in $G \cap \mathcal{W}_{\gamma}^{s}(x)$. Since $y_{1}, y_{2} \in B_{0}$, the Hopf argument (see Lemma 3.3) shows that $\xi\left(\psi\left(y_{1}\right)\right)=\xi\left(\psi\left(y_{2}\right)\right)$. Let $m$ denote this ergodic measure. Since $y_{1} \in B_{K}, m(K)=1$ so that there is a large enough $j$ for which $m\left(K_{j}\right)>\frac{1}{2}$. Since $y_{1}, y_{2} \in B_{\chi\left(K_{j}\right)}$ there are infinitely many $n$ for which $\gamma^{n} y_{1}$ and $\gamma^{n} y_{2}$ both lie in $K_{j}$. Let $\epsilon>0$. As $\gamma$ contracts distances in $\mathcal{W}_{\gamma}^{s}(x)$ by some constant $\lambda<1$ and by uniform continuity of $\sigma$ in $K_{j}$, there is a large enough $N$ so that $d_{C}\left(\sigma\left(\gamma^{N} y_{1}\right), \sigma\left(\gamma^{N} y_{2}\right)\right)<\epsilon$. By iterating $(* * *)$, we therefore obtain that

$$
\begin{gathered}
d_{C}\left(\sigma\left(y_{1}\right), \sigma\left(y_{2}\right)\right)=\left\|\sum_{i=0}^{N-1} \widehat{\gamma^{i}}\left(f\left(\gamma^{i} y_{1}\right)-f\left(\gamma^{i} y_{2}\right)\right)+\left(\sigma\left(\gamma^{N} y_{1}\right)-\sigma\left(\gamma^{N} y_{2}\right)\right)\right\| \\
\leq \sum_{i=0}^{N-1} L d_{M}\left(\gamma^{i} y_{1}, \gamma^{i} y_{2}\right)+\epsilon \leq \sum_{i=0}^{\infty} L \lambda^{i} d_{M}\left(y_{1}, y_{2}\right)+\epsilon
\end{gathered}
$$


where $L$ is a Lipshitz constant for $f$ and $\lambda$ is the contraction constant for $\gamma$.

Finally, we show that the map $\phi: \mathbb{R}^{n} \rightarrow C$ defined by $x \mapsto \operatorname{proj}_{C}(x)+\sigma([x])$ is equivariant:

$$
\begin{gathered}
\phi(\bar{\rho}(\gamma) x)=\operatorname{proj}_{C}(\bar{\rho}(\gamma) x)+\sigma(\rho(\gamma)[x])=\operatorname{proj}_{C}(\pi(\gamma) x+\pi(\gamma) A(\gamma,[x]))+\sigma(\rho(\gamma)[x])= \\
\pi^{C}(\gamma) \operatorname{proj}_{C}(x)+\pi_{C}(\gamma) A^{C}(\gamma,[x])+\sigma(\rho(\gamma)[x])=\pi^{C}(\gamma) \operatorname{proj}_{C}(x)+\pi^{C}(\gamma) \sigma([x])= \\
\pi^{C}(\gamma) \phi(x),
\end{gathered}
$$

where the penultimate equality follows from $(* *)$.

Lemma 4.2. With the same hypotheses as in Prop 4.1, the map $\phi$ is constant.

Proof. Fix a lift $\widetilde{\mathcal{W}}^{-}(x)$ of a stable leaf of a partially hyperbolic diffeomorphism $\rho(\gamma)$ and let $y \in \widetilde{\mathcal{W}}^{-}(x)$. Suppose that $d_{0}:=d_{C}(\phi(x), \phi(y))>0$. By uniform continuity of $\phi$ there is a $\delta>0$ so that $d(x, y)<\delta$ implies $d_{C}(\sigma(x), \sigma(y))<\frac{d_{0}}{2}$. For sufficiently large $n, d\left(\bar{\rho}\left(\gamma^{n}\right) x, \bar{\rho}\left(\gamma^{n}\right) y\right)<\delta$. Therefore,

$\left.d_{0}=d_{C}(\phi(x), \phi(y))=d_{C}\left(\pi^{C}\left(\gamma^{n}\right) \phi(x), \pi^{C}\left(\gamma^{n}\right) \phi(y)\right)\right)=d_{C}\left(\phi\left(\bar{\rho}\left(\gamma^{n}\right) x\right), \phi\left(\bar{\rho}\left(\gamma^{n}\right) y\right)\right)<\frac{d_{0}}{2}$.

This yields a contradiction unless $\phi$ is constant on lifts of stable leaves and therefore locally constant by the local accessibility property for weakly hyperbolic families.

Theorem 4.3. With the same hypotheses as in Prop 4.1, the subspace $C$ is trivial.

Proof. Suppose not. Since $\phi$ is defined by adding a bounded map to the projection map to the subspace $C, \phi$ is unbounded, contradicting Lemma 4.2.

\section{Towards Global Rigidity}

In this section we assume that the acting group $\Gamma$ is a lattice in a connected semisimple real Lie group $G$ with each (almost) simple factor having real rank at least two. For simplicity we call such a group a higher rank lattice. In this section, we deduce that all weakly hyperbolic $C^{2}$ volume preserving actions on a torus that lift to the universal cover are semiconjugate to the linear action coming from the fundamental group when restricted to a finite index subgroup of the acting lattice. We also argue that this semiconjugacy is injective under the additional hypothesis that the leaves of the lift of the unstable foliation of a partially hyperbolic group element are quasiisometrically embedded in $\mathbb{R}^{n}$. 
Let $\rho: \Gamma \rightarrow \operatorname{Diff}^{2}\left(\mathbb{T}^{n}\right)$ be a volume preserving action that lifts to an action on the universal cover, $\bar{\rho}: \Gamma \rightarrow \operatorname{Diff}^{2}\left(\mathbb{R}^{n}\right)$. Let $\pi_{\rho}: \Gamma \rightarrow \mathrm{GL}(n, \mathbb{Z})$ be the induced homomorphism and $\rho_{0}$ be the affine action induced by $\pi_{\rho}$. In $[\mathrm{MQ}$, they make the following:

Definition. The representation $\pi_{\rho}: \Gamma \rightarrow \mathrm{GL}(n, \mathbb{R})$ is said to be weakly hyperbolic if the Zariski closure of $\pi(\Gamma) \subset \mathrm{GL}\left(n, \mathbb{R}^{n}\right)$ is not precompact in any of its nontrivial subrepresentations.

The next theorem is from $\mathbb{M Q}$. We remark that although they assume the existence of a periodic point for the action, their proof works equally well under the weaker assumption that the action lifts to $\mathbb{R}^{n}$.

Theorem 5.1. $\left[M Q\right.$, Theorem 6.10] Let $\Gamma, \rho$, and $\bar{\rho}$ be as above. If $\pi_{\rho}$ is weakly hyperbolic, then there exists a finite index subgroup $\Gamma^{\prime}<\Gamma$ and a map $\phi \in C^{0}\left(\mathbb{T}^{n}\right)$, unique in the homotopy class of the identity such that $\phi \circ \rho(\gamma)=\rho_{0}(\gamma) \circ \phi$ for all $\gamma \in \Gamma^{\prime}$.

Next we establish a complimentary statement.

Theorem 5.2. Let $\Gamma, \rho$, and $\bar{\rho}$ be as above. If $\rho$ is weakly hyperbolic, then $\pi_{\rho}$ is weakly hyperbolic.

Proof. Suppose that the Zariski closure of $\pi(\Gamma)$ is precompact in $\mathrm{GL}(C)$ for some invariant subspace $C$. Since the Zariski closure of $\pi(\Gamma)$ is semisimple (Mar, Prop. IX 5.7], there is an invariant subspace complementary to $C$. Since $\Gamma$ has property (T), Theorem 4.1 implies $C=0$.

Corollary 5.3. Let $\Gamma, \rho$, and $\bar{\rho}$ be as above. If $\rho$ is weakly hyperbolic, then after passing to a finite index subgroup of $\Gamma, \rho$ is $C^{0}$-semiconjugate to the affine action coming from the homomorphism $\Gamma \rightarrow \operatorname{Out}\left(\pi_{1}\left(\mathbb{T}^{n}\right)\right)$ by a map which is unique in the homotopy class of the identity.

Remark: For many lattices, the above results hold without assuming that the lattice action $\rho$ on the torus is covered by an action $\bar{\rho}$ of $\mathbb{R}^{n}$. Indeed, the action will lift whenever $H^{2}\left(\Gamma, \mathbb{Z}^{n}\right)=0$ (see e.g. [FW]). For relevant cohomology vanishing results see $[\mathrm{Bo}], \mathrm{BoW}],[\mathrm{Zu}$, and $[\mathrm{K}]$.

Next we argue that this semiconjugacy is a $C^{0}$ conjugacy provided that the lifts of unstable leaves to $\mathbb{R}^{n}$ have intrinsic distances comparable to Euclidean distance for some partially hyperbolic group element. Following [B2] we make the following: 
Definition. A foliation $\mathcal{W}$ of a simply connected metric space $(X, d)$ is said to be quasi-isometric if there are uniform constants $a, b>0$ such that for each $x \in X$ and $y \in \mathcal{W}(x), d_{\mathcal{W}(x)}(x, y) \leq a d_{X}(x, y)+b$.

Proposition 5.4. Let $\Gamma, \rho, \bar{\rho}, \pi$, and $\rho_{0}$ be as above. Let $\phi$ be the unique continuous map homotopic to the identity such that $\phi \circ \rho(\gamma)=\rho_{0}(\gamma) \circ \phi$ for all $\gamma \in \Gamma$. If there exists a group element $\gamma \in \Gamma$ so that $\rho(\gamma)$ is partially hyperbolic and so that the lift $\widetilde{\mathcal{W}}_{\rho(\gamma)}^{u}$ of the unstable foliation $\widetilde{\mathcal{W}}_{\rho(\gamma)}^{u}$ to $\mathbb{R}^{n}$ is quasi-isometric, then $\phi$ is a homeomorphism.

Proof. The map $\phi$ has degree one and is therefore surjective. To prove injectivity, it suffices to prove that $\phi$ is locally injective. It therefore suffices to show that a lift of $\phi$ to $\mathbb{R}^{n}$ is locally injective. First we argue that $\phi$ may be equivariantly lifted with respect to $\bar{\rho}$ and $\pi$. Choose a lift $\tau: \mathbb{R}^{n} \rightarrow \mathbb{R}^{n}$ of $\phi$ and define

$$
\theta: \Gamma \times \mathbb{R}^{n} \rightarrow \mathbb{R}^{n}
$$

by $\theta(\gamma, x)=\tau(\bar{\rho}(\gamma)(x))-\pi(\gamma)(\tau(x))$. Since $\tau(\bar{\rho}(\gamma)(x))$ and $\pi(\gamma)(\tau(x))$ project to the same point in the torus, $\theta\left(\Gamma \times \mathbb{R}^{n}\right) \subset \mathbb{Z}^{n}$. As $\mathbb{Z}^{n}$ is discrete, each $\theta(\gamma, \cdot)$ is constant so that we may alternatively view $\theta$ as a map

$$
\theta: \Gamma \rightarrow \mathbb{Z}^{n} \subset \mathbb{R}^{n} .
$$

Viewed this way, $\theta$ is a one cocycle over $\pi$. Indeed,

$$
\begin{gathered}
\theta\left(\gamma_{1} \gamma_{2}\right)=\tau\left(\bar{\rho}\left(\gamma_{1} \gamma_{2}\right)(0)\right)-\pi\left(\gamma_{1} \gamma_{2}\right)(\tau(0))= \\
\tau\left(\bar{\rho}\left(\gamma_{1}\right)\left(\bar{\rho}\left(\gamma_{2}\right)(0)\right)\right)-\pi\left(\gamma_{1}\right)\left(\pi\left(\gamma_{2}\right)(\tau(0))\right)= \\
\theta\left(\gamma_{1}, \bar{\rho}\left(\gamma_{2}\right)(0)\right)+\pi\left(\gamma_{1}\right)\left(\tau\left(\bar{\rho}\left(\gamma_{2}\right)(0)\right)\right)-\pi\left(\gamma_{1}\right)\left(\pi\left(\gamma_{2}\right)(\tau(0))\right)= \\
\theta\left(\gamma_{1}\right)+\pi\left(\gamma_{1}\right)\left(\theta\left(\gamma_{2}\right)\right),
\end{gathered}
$$

for each $\gamma_{1}, \gamma_{2} \in \Gamma$. Since $H^{1}(\Gamma, \pi)=0$ ([프, Theorem 2]), there is a $v \in \mathbb{R}^{n}$ so that

$$
\theta(\gamma)=\pi(\gamma)(v)-v
$$

for each $\gamma \in \Gamma$. Define

$$
\bar{\phi}: \mathbb{R}^{n} \rightarrow \mathbb{R}^{n}
$$

by $\bar{\phi}(x)=\tau(x)+v$. It is straightforward to check that $\bar{\phi}$ is equivariant with respect to $\bar{\rho}$ and $\pi$. It therefore remains to show $\bar{\phi}$ is a cover of $\phi$. First note that $\bar{\phi}$ descends to a map $\phi^{\prime}$ homotopic to the identity on $\mathbb{T}^{n}$ since

$$
\bar{\phi}(x+z)=\tau(x+z)+v=\tau(x)+z+v=\bar{\phi}(x)+z,
$$


for each $x \in \mathbb{R}^{n}$ and $z \in \mathbb{Z}^{n}$. Moreover, $\phi^{\prime}$ is equivariant with respect to $\rho$ and $\rho_{0}$ and therefore coincides with $\phi$ by uniqueness.

To finish the argument, we show that $\bar{\phi}$ is locally injective. Since $\phi$ is homotopic to the identity there is some $M>0$ so that $\|\bar{\phi}(x)-x\|<M$. If $\bar{\phi}$ is not locally injective, we may choose $x, y \in \mathbb{R}^{n}$ with the same image and sufficiently close so that there exists a piecewise $C^{1}$ curve $\sigma:[0,1] \rightarrow \mathbb{R}^{n}$ satisfying $\sigma(0)=x, \sigma(1) \in \widetilde{\mathcal{W}}_{\rho(\gamma)}^{u}(y)$, and $\dot{\sigma} \in E_{\rho(\gamma)}^{s} \oplus E_{\rho(\gamma)}^{c}$. By equivariance, $\bar{\phi}\left(\bar{\rho}\left(\gamma^{n}\right)(x)\right)=\bar{\phi}\left(\bar{\rho}\left(\gamma^{n}\right)(y)\right)$ for each natural number $n$. Therefore,

$$
\begin{gathered}
2 M>\left\|\bar{\rho}\left(\gamma^{n}\right) x-\bar{\rho}\left(\gamma^{n}\right) y\right\| \geq\left\|\bar{\rho}\left(\gamma^{n}\right) y-\bar{\rho}\left(\gamma^{n}\right) \sigma(1)\right\|-\left\|\bar{\rho}\left(\gamma^{n}\right) \sigma(1)-\bar{\rho}\left(\gamma^{n}\right) x\right\| \\
\geq \frac{1}{a} d_{\widetilde{\mathcal{W}}_{\gamma}^{u}}\left(\bar{\rho}\left(\gamma^{n}\right) \sigma(1), \bar{\rho}\left(\gamma^{n}\right) y\right)-K \operatorname{length}\left(\bar{\rho}\left(\gamma^{n}\right) \sigma\right)-\frac{b}{a},
\end{gathered}
$$

a contradiction since the last term of this inequality grows unbounded with $n$.

\section{References}

[A] D.V. Anosov. Geodesic flows on closed Riemannian manifolds of negative curvature. Proc. Steklov. Inst. Math. 90 (1967).

[Bo] A. Borel. Stable real cohomology of arithmetic groups II. Prog. in Math. 14 (1981).

$[\mathrm{BoW}]$ A. Borel and W. Wallach. Continuous cohomology, discrete subgroups, and representations of reductive groups, Annals of Mathematics Studies 94, Princeton University Press 1980.

[B1] M. Brin. Ergodicity of geodesic flows. Appendix in: Lectures on Spaces of Nonpositive Curvature, Ballmann Birkhauser, 1995.

[B2] M. Brin. On dynamical coherence. Ergodic Theory Dynam. Systems 23(2) (2003) 395-401.

[BP] M. Brin and Ja. Pesin. Partially hyperbolic dynamical systems. Math. USSR Izvestija 8(1974). 177-218.

[BPSW] K. Burns, C. Pugh, M. Shub, and A. Wilkinson. Recent results about stable ergodicity. Proc. Symposia A.M.S. 69 (2001) 327-366.

[D] P. Delorme. 1-cohomologie des representations unitaires des groupes de Lie semisimples et resolubles. Produits tensoriels continus representations, Bull. Soc. Math. France 105 (1977), 281-336. 
[FW] D. Fisher and K. Whyte. Continuous quotients for lattice actions on compact spaces. Geom. Ded. 87 (2001) 181-189.

[FMW] D. Fisher, D. Morris, K. Whyte. Non-ergodic actions, cocycles and superrigidity, to appear New York Journal Math.

[F] J. Franks. Anosov diffeomorphisims. Global Analysis (Proceedings of the Symposia in Pure Mathematics, 14). American Mathematical Society, Providence, RI, (1970), 61-93.

[G] A. Guichardet. Cohomologie des groupes topologiques et des algebres de Lie. F. Nathan-Cedic, 1980.

[HPS] M. Hirsch, C. Pugh, M. Shub. Invariant Manifolds (Lecture Notes in Mathematics, 583). Springer, 1977.

[HuK] S. Hurder and A. Katok. Differentiability, rigidity, and Godbillon-Vey classes for Anosov flows. Publ. Math. Inst. Hautes Etudes Sci. 72 (1990), 5-61.

[J] J.-L. Journe. A regularity result lemma for functions of several variables. Revista Matematica Iberoamericana 4(2) (1988), 187-193.

[KP] W. Kryszewski and S. Plaskacz. Topological methods for the local controllability of nonlinear systems. SIAM J. Control and Optimization 32(1) (1994), 213-223.

[K] S. Kumaresan. On the canonical k-types in the irreducible unitary g-modules with non-zero relative cohomology. Inventiones math., 59, (1980) 1-11.

[L] R. de la Llave. Remarks on Sobolev regularity in Anosov systems. Erogodic Theory and Dynam. Systems 21 (2001) no.4, 1139-1180.

[LMM] R. de la Llave, J. M. Marco, R. Moryion. Canonical perturbation theory of Anosov systems and regularity results for Livsic cohomology equation. Ann. Math. 123 (1986), 537-612.

[Ma] A. Manning. There are no new Anosov diffeomorphisms on tori. Amer. J. Math. 96(3) (1974), 422-429.

[Mar] G. Margulis. Discrete subgroups of semisimple Lie groups. Springer, New York, 1991.

[MQ] G. Margulis and N. Qian. Rigidity of weakly hyperbolic actions of higher real rank semisimple Lie groups and their lattices. Ergod. Th. and Dynam. Sys. (2001), 21, 121-164. 
[Mi] J. Milnor. Fubini foiled: Katok's paradoxical example in measure theory. Math. Intelligencer, 19 (1997), no.2, 30-32.

[NT] V. Nitica and A. Torok. Regularity of the transfer map for cohomologous cocycles. Ergod. Th. and Dynam. Sys. (1998) (18), 1187-1209.

[P] Ya. B. Pesin. On the existence of invariant fibering for the diffeomorphisms of a smooth manifold. Math. USSR Sbornik 20(2) (1973), 213-222.

[PS] C. Pugh and M. Shub. Ergodicity of Anosov actions. Invent. Math. 15 (1972), $1-23$.

[RT] J. Rauch and M. Taylor. Regularity of functions smooth along foliations, and elliptic regularity. Journal of Functional Analysis, 225 (2005), 74-93.

[S] A.N. Starkov. Vanishiing of the first cohomologies for lattices in Lie groups. Journal of Lie Theory, 12 (2002), 449-460.

[T] M. Taylor. Partial Differential Equations III. Applied Mathematical Sciences (Springer-Verlag New York Inc. 117

[Z] R.J. Zimmer. Actions of semisimple groups and discrete subgroups, Proc. Internat. Cong. Math, Berkeley, 1986, 1247-1258.

[Zu] G. Zuckerman. Continuous cohomology and unitary representations of real reductive groups. Annals of Mathematics, 107 (1978), 495-516. 